\title{
Retrospective and spatial analysis tools for integrated surveillance of cystic echinococcosis and bovine cysticercosis in hypo-endemic areas
}

\author{
Rudi Cassini ${ }^{1}$, Paolo Mulatti ${ }^{2}$, Claudia Zanardello², Giulia Simonato ${ }^{1}$, Manuela Signorini ${ }^{1}$,

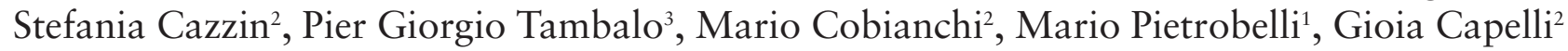 \\ ${ }^{1}$ Department of Animal Medicine, Production and Health, University of Padova, Legnaro, Italy; ${ }^{2}$ Istituto \\ Zooprofilattico Sperimentale delle Venezie, Legnaro, Italy; ${ }^{3}$ Local Health Unit, Caprino Veronese, Italy
}

\begin{abstract}
Cystic echinococcosis (CE) and bovine cysticercosis (BC) are two important parasitic zoonoses, whose prevalence varies among European countries. Few data are available on prevalence and geographic distribution of these two diseases in Veneto region in north-eastern Italy, where they are generally perceived as minor public health problems. Available data from regional farms on cattle positive to CE and BC and slaughtered in the period 2006-2010 were analysed by spatial scan statistic using a Bernoulli probability model. Out of 576 bovines testing positive to CE, 467 were found to be autochthonous cases. Three significant CE clusters were identified, the most likely one $(\mathrm{P}<0.001)$ located in the eastern part of the Veneto region. As for BC, two clusters were identified from 148 animals testing positive, 91 of which were autochthonous. An epidemiological survey was conducted and the most likely CE cluster was centred, collecting faecal samples from 28 dogs living in the farms of the area. Out of five animals (all shepherd dogs) found positive for taenid eggs by copromicroscopy, one was confirmed positive for Echinococcus granulosus by means of polymerase chain reaction. The study demonstrates the usefulness of integration of slaughterhouse data and geographical coordinates of farms involved for effective surveillance of $\mathrm{CE}$ and BC. The reliability of the spatial analysis in the identification of clusters of EC cases was confirmed by the finding of one dog positive for E. granulosus.
\end{abstract}

Keywords: bovine cysticercosis, cystic echinococcosis, integrated surveillance, retrospective analysis, spatial analysis, Italy.

\section{Introduction}

Cattle act as intermediate hosts for both Echinococcus granulosus and Taenia saginata, two species of tapeworm causing important zoonotic, parasitic diseases. E. granulosus is the aetiological agent of cystic echinococcosis (CE), a generally non-pathogenic infection in dogs (the definitive host) but a serious and potentially lethal disease in humans, who act as intermediate hosts harbouring the larval stage (hydatid cyst). Many domestic and wild mammals may also act as intermediate hosts with sheep playing the central role in maintaining CE in Italy. Areas of central and southern Italy, where sheep herds are usually bred with traditional methods, show high prevalence values (Garippa and Manfredi, 2009). In contrast, CE is rarely reported in regions with marginal sheep breeding and scarce ovine populations such as in

\section{Corresponding author:}

Rudi Cassini

Department of Animal Medicine, Production and Health

University of Padova

Viale dell'Università, 16 - 35020 Legnaro (PD), Italy

Tel. +39049 827-2777; Fax +39049 827-2794

E-mail: rudi.cassini@unipd.it northern Italy, where the prevalence in cattle is generally below $1 \%$ as per data recorded during different retrospective studies at slaughterhouse (Garippa and Manfredi, 2009). The island of Sardinia, on the other hand, is hyper-endemic with prevalence values higher than $30 \%$ recorded in cattle between 2 and 6 years old and up to $80 \%$ in older animals (Scala et al., 2004a).

The adult phase of T. saginata is responsible for taeniasis in humans and the larval stage causes bovine cysticercosis (BC) in cattle. The prevalence of BC, usually based on abattoir survey data, ranges from $0.007 \%$ to $6.8 \%$ in European countries. In Italy, prevalence values among the Italian regions differ only within the low range of $0.02 \%$ to $2.4 \%$ (European Commission, 2000). The incidence of human taeniasis is difficult to calculate since it is not a notifiable disease. An estimate can be obtained using sales figures of specific antiparasitic drugs in humans, which suggest prevalence rates between $0.02 \%$ and $0.62 \%$ in western Europe (European Commission, 2000).

According to the current European Union (EU) meat inspection regulation (EC) no. 854/2004, all bovines over 6 weeks of age have to be individually inspected for $\mathrm{BC}$ at slaughter. In addition, according to the European Directive no. 2003/99/EC, the echinococco- 
sis parasite belongs to the zoonotic, infectious agents for which monitoring shall take place at the most appropriate stage of the food chain in all member countries. In Italy, the veterinary officer carrying out the post-mortem inspection shall register the identification number of all cattle found positive for $\mathrm{CE}$ or $\mathrm{BC}$ and report this information to the local health unit (ULSS - using the Italian acronym) in charge of veterinary services in the area of the positive farm. The ULSS staff is supposed to autonomously implement further investigation.

In Europe, prevalence and other characteristics of $\mathrm{CE}$ have been investigated by various scientists in different bovine populations using samples and data collected at the abattoir (Scala et al., 2004a; Rinaldi et al., 2008; Umhang et al., 2013) and the same is true for BC (Boone et al., 2007; Allepuz et al., 2012; Calvo-Artavia et al., 2013; Dupuy et al., 2013). Only recently geospatial tools have been applied for the study of these two diseases (Guazzetti et al., 2006; Cringoli et al., 2007; Allepuz et al., 2009; Manfredi et al., 2011; Mastin et al., 2011; Brundu et al., 2012).

$\mathrm{CE}$ and $\mathrm{BC}$ are generally not perceived as major problems in all areas of north-eastern Italy and only scattered information is available on prevalence, geographical distribution and risk factors associated with these parasites. The present study reports the results of a project funded by the Veneto region (DGRV 2221/2010) aimed at collection, organisation and spatial analysis of available data on local farm cattle slaughtered in the period 2006-2010 and found positive to $\mathrm{CE}$ and/or BC. In order to identify areas of major risk, retrospective, spatial analysis was carried out with special reference to the detection of likely clusters of autochthonous cases among the regional bovine populations.

\section{Materials and methods}

\section{Data sources}

The study was limited to the Veneto region in northeastern Italy, which covers an area of $18,390 \mathrm{~km}^{2}$ divided into seven provinces. In 2007, a total of 16,007 active cattle farms $(966,893$ heads) were registred. Both dairy and beef production represent an important economic sector among primary production activities of the region. In contrast, sheep and goat rearing is remarkably less important and only 1,341 sheep farms (27,716 heads) and 919 goat farms (7,366 heads) were recorded at this time. Data on animal populations, farms and the total numbers of slaugh- tered animals are available at: http://agri.istat.it/ jsp/Introduzione.jsp?id=8A from of the Italian National Institute of Statistics (ISTAT). Slaughtered catthe are aggregated into four groups according to the national classification: calves (6-12 months of age), baby beefs (12-24 months of age), bulls (male bovines older than 24 months) and cows or dairy cattle (female bovines older than 24 months). According to the ISTAT data, a total amount of 4,723,021 bovines coming from Veneto region farms were slaughtered in the period 2006-2010; precisely $1,554,700$ calves, $2,985,262$ baby beefs, 40,571 bulls and 142,488 dairy cattle.

The geographical information systems (GIS) office of the Istituto Zooprofilattico Sperimentale delle Venezie records the geographical coordinates for all bovine farms (Ferrè et al., 2011) in the region. These data are integrated with information on the health status of the farms and saved in a geo-relational database. Since all bovine farms are registered with a specific code (ID) in the national bovine identification database (BDN) according to regulation (EC) no. 1760/2000, farms positive for CE/BC infection could be extracted from the database.

All 21 ULSS of the Veneto region are requested to provide identification tags of cattle positive to $\mathrm{CE}$ and/or BC coming from their territory in the period 2006-2010. Identification tags were used to track provenance and movements of the animals. Animals with an incomplete movement list (mainly the ones born outside Italy) were excluded from the study. Positive cases were considered autochthonous if they were born and never moved from Veneto region. Only farms where at least one autochthonous case was identified were considered in the spatial analyses.

\section{Spatial analysis}

The spatial aggregation of positive farms was investigated through a purely spatial scan statistic, using a Bernoulli probability model (Kulldorff, 1997), which is particularly suitable for presence/absence or positive/negative data. As previously mentioned, positive farms were considered those farms where the detected $\mathrm{CE} / \mathrm{BC}$ case surely acquired the infection, spending all its life on the farm. All other farms were considered negative. The method consists in scanning the study area by placing a number of circles (spatial windows) with increasing radius over the area, and counting the number of positive and/or negative events occurring in the spatial window (Kulldorff, 1997). Only dairy farms were included in the control population, since positive animals were only found in this type of farm. 
A total of 8,173 active dairy farms existed in 2010 (BDN database) and were therefore considered as the reference population of our study. The maximum size of the spatial scanning window was set to include up to $3 \%$ of the total population of dairy cattle farms for $\mathrm{CE}$ and $1 \%$ for $\mathrm{BC}$, accordingly to the overall low proportion of positive farms on the whole bovine farms population considered (251 and 67 observed cases for $\mathrm{CE}$ and $\mathrm{BC}$, respectively).

The likelihood function was calculated for each circular zone by comparing the number of $\mathrm{CE}$ and $\mathrm{BC}$ cases to the expected number of cases in the population at risk, based on the number of farms falling inside the spatial windows and the proportion of cases vis-à-vis control farms. A Monte Carlo hypothesis testing approach was then used to assess the statistical significance of the pattern of points observed, assuming that $\mathrm{CE} / \mathrm{BC}$ cases were a random sample of the total number of tested farms. A cluster of positive farms was considered significant when the simulated P-value was $\leq 0.05$ (Dwass, 1957). The identified clusters were ranked on the basis of a likelihood function (Kulldorff and Nagarwalla, 1995; Kulldorff, 1997). The spatial window with the maximum number of cases was classified as the most likely cluster; other significant clusters were classified as secondary, while the least likely clusters were deemed to have occurred by chance. Further data obtained were the geographical coordinates of the centroid, the radius of the spatial window, the number of observed and expected positive farms, and the total population in the cluster. The data were handled by SaTScan ${ }^{\mathrm{TM}}$ version 9.1.1 (http://www.satscan.org/), while the output was visualised in ESRITM ArcMap ${ }^{\circledR}$ version 10.1 (http://www.esri.com/).

\section{Copromicroscopic survey in dogs}

In June 2012, an epidemiological survey, based on dog faecal samples, was conducted in the most likely CE cluster, identified in the municipality of San Zeno di Montagna in north-western Verona province. All farm dogs from dairy and sheep farms located in the cluster area, whose owners were willing to collaborate, were included in the survey. The aim of the survey was to detect at least one positive case, if the infection was present in the dog population. Individual samples were retrieved from 28 dogs, belonging to eight bovine farms with positive cases of CE, three negative dairy cattle farm and to two sheep farms sharing grazing areas with the positive farms.

All samples were frozen at $-80^{\circ} \mathrm{C}$ for at least 72 hours to ensure any viable eggs were killed prior to examina- tion, then thawed, divided in two aliquots of $2 \mathrm{~g}$ each, diluted with approximately $8 \mathrm{ml}$ of tap water and thoroughly mixed prior to centrifugation at $1600 \times g$ for 10 $\mathrm{min}$. The first aliquot was then submitted to the egg isolation procedure described by Davidson et al. (2009) and the second subjected to copromicroscopic examination with flotation technique using a zinc chloride solution (specific weight 1450). If faecal material was insufficient, only the isolation procedure was performed. The presence of taeniid eggs was detected by means of an inverted microscope in a $10 \mathrm{ml}$ closed tube with flattened side, from which the liquid obtained at the end of the isolation procedure was carefully poured into a $15-\mathrm{ml}$ conical Falcon ${ }^{\circledR}$ centrifuge tube. Positive samples were investigated by polymerase chain reaction (PCR) (Trachsel et al., 2007) after further sedimentation for $30 \mathrm{~min}$, and aspiration of excess fluid from the tube, in order to ensure a final volume of about $0.5 \mathrm{ml}$. The PCR results were confirmed by the National Reference Centre for Echinococcus granulosus (CeNRE, IZS Sardinia).

\section{Results}

\section{Cystic echinococcosis}

Overall, 576 bovines tested positive for CE and 467 $(81.1 \%)$ were reliably identified as autochthonous cases. With the exception of two baby beefs, all positive cases were dairy cattle. Consequently, the prevalence value of the disease in Veneto region was calculated as the ratio between the number of autochthonous cases and the total number of slaughtered dairy cattle $(n=142,488)$ in the period 2006-2010. Therefore, the estimated prevalence was $0.33 \% \quad(95 \%$ confidence interval $(\mathrm{CI})=0.30-0.36 \%)$. One of the baby beefs was female, while the gender of the other is unknown. However, the estimated prevalence remains $0.33 \%$, regardless if this is included in the calculation or not.

For 395 of the positive animals it was possible to certainly identify the farm where the infection occurred, and only these positive farms $(\mathrm{n}=251)$ were considered in the spatial analysis (Fig. 1a). The number of positive animals per farm ranged from 1 to 13 . Spatial analysis identified three significant clusters of $\mathrm{CE}$ and the most likely one $(\mathrm{P}<0.001)$ situated in the north-western part of Verona province (Fig. 2; Table 1).

\section{Bovine cysticercosis}

Out of 148 positive animals, $91(61.5 \%)$ were considered autochthonous. All positive cases were dairy cattle and, using the same approach applied for CE, 

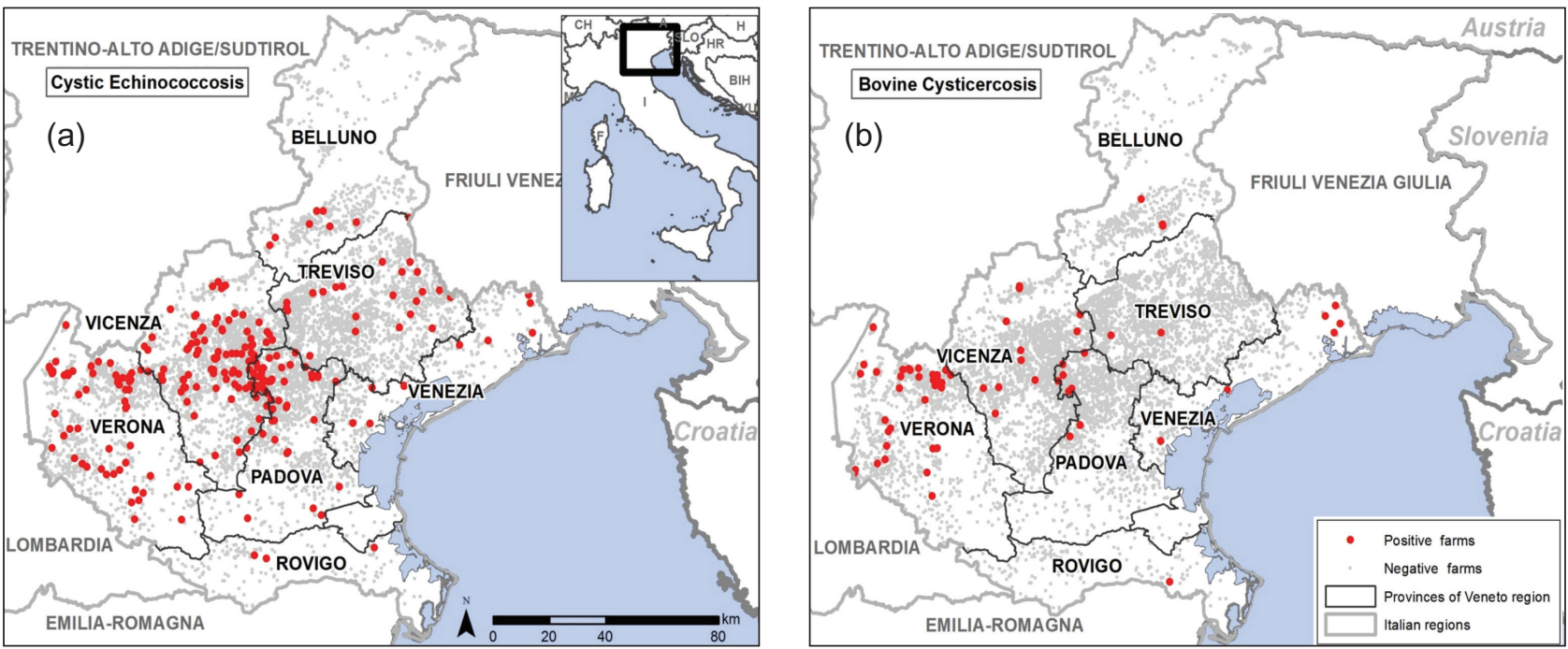

Fig. 1. Map of the Veneto region with dairy farms testing negative (grey dots) for CE (a) and farms testing positive (red dots) for $\mathrm{BC}(\mathrm{b})$.

the estimated prevalence resulted in $0.06 \%$ with a $95 \%$ CI of $0.05-0.08 \%$. The spatial scan statistic included 67 positive farms (Fig. 1b) identified as the certain infection source for 74 of the animals. A maximum of two positive individuals per farm were detected. Between the two clusters identified by spatial analysis, the most likely one $(\mathrm{P}<0.01)$ was found in the north-eastern part of Verona province (Fig. 3; Table 2).

\section{Presence of taenid eggs in dogs}

The copromicroscopic examination with sedimentation and flotation technique was not performed for seven samples because of scarce material. Taenid eggs were found only in one sample. The isolation procedure was performed for all 28 samples and taenid eggs were detected in five of them $(17.9 \%)$. The isolation procedure confirmed the results of the copromicroscopic technique and in addition identified cestode eggs in one sample negative at copromicroscopy, whereas the other three positive samples were only examined by this procedure. All the five positive animals were shepherd dogs belonging to two farms. In one farm ( $3 / 3$ positive dogs) sheep breeding still follow the traditional transhumant system, i.e. movement of people with their livestock between fixed summer and winter pastures. In the other farm (2/4 positive dogs) sheep and cattle are reared together with the sheep flock generally confined to the farm area. Only one sample out of the five analysed by PCR analysis, tested positive for E. granulosus, corresponding to one of the three positive dogs of the traditional transhumant sheep flock.

\section{Discussion}

The prevalence of CE in the bovine population of Veneto region estimated in the present study is similar to what is encountered in other northern regions of Italy (Garippa and Manfredi, 2009; Manfredi et al., 2011). BC was also found to exist at low prevalence in the intermediate host. These results confirm the hypoendemic status of these two diseases in the region, while the finding of three significant clusters for CE, and two for BC, confirms the initial hypothesis of aggregated distribution of the two parasites. A detailed investigation of positive farms in the cluster areas could facilitate identification of the most likely routes of introduction of the parasites into the bovine

Table 1. Characteristics of CE clusters.

\begin{tabular}{lccccc}
\hline Cluster ID & Radius $(\mathrm{m})$ & No. of farms included & No. of cases observed & No. of cases expected & P-value \\
\hline 1 & 1,333 & 18 & 10 & 0.56 & $<0.001$ \\
2 & 4,219 & 198 & 22 & 6.08 & 0.01 \\
3 & 1,384 & 7 & 5 & 0.22 & 0.012 \\
\hline
\end{tabular}




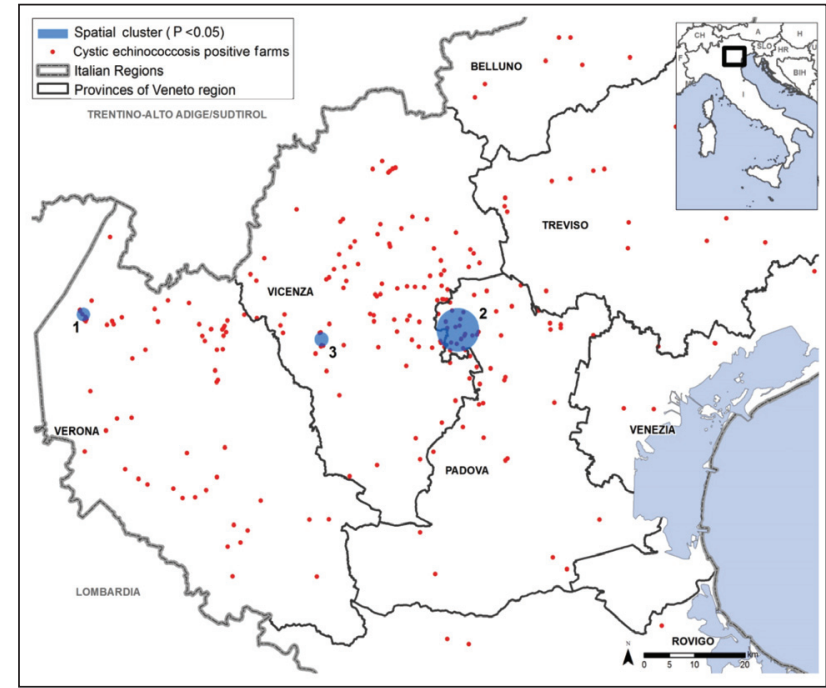

Fig. 2. Geographical localization of CE clusters in the Veneto region.

farms and consequently elucidate the risk factors associated with the maintenance and spreading of these diseases.

With regard to CE, the presence of free-ranging dogs and transhumant sheep farming is probably maintaining the life cycle and consequently represents the origin of the infection for the bovines (Scala et al., 2004b). However, animal production systems found in the two likely clusters were different (cluster 1 was found in a mountainous area highly used for grazing and cluster 2 in a plane area with animals, mostly confined) and the epidemiological features of the disease may change consequently. Concerning $\mathrm{BC}$, the use of pasture is a known risk factor, but many other routes of introduction have to be considered, such as the use of contaminated water or the presence of infected staff on the farm (European Commission, 2000). In the present study, the more likely cluster was found in a mountainous grazing area, where cattle are often left to graze and the use of surface drinking water for animals is frequent.

Interestingly, the present study demonstrates the usefulness of gathering and integration of data from different sources (slaughterhouses, passive surveillance, the BDN database, geographical coordinates of farms) for effective support in the epidemiological analysis. In

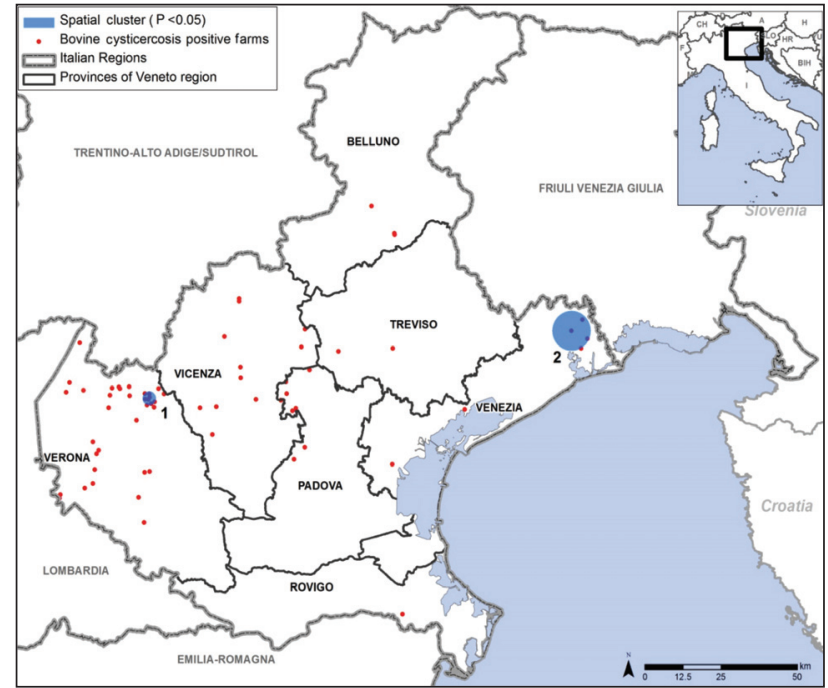

Fig. 3. Geographical localization of BC clusters in the Veneto region.

particular, we recommend this approach for these two parasitic diseases, whose diagnosis is mandatory and can easily be implemented for all slaughtered bovines in Italy. In our case, the reliability of the identification of an EC cluster in a small area was confirmed by one dog found positive to E. granulosus.

GIS and spatial analyses constitute a useful approach that supports the generation of hypotheses on drivers for disease diffusion based on geographical inter-relationships (or constraints) between host populations and environmental determinants (Pfeiffer et al., 2008). In our study, spatial analysis contributed strongly to elucidating distribution and aggregation of cases of the bovine farms with cases of CE and/or BC. As far as we know, only a few European studies (Guazzetti et al., 2006; Allepuz et al., 2009; Brundu et al., 2012) have focused on GIS and/or spatial analysis tools with regard to the epidemiology of these two infections based on retrospective data obtained through passive slaughterhouse surveillance systems. Bovine is the animal of choice in this type of analysis, for $\mathrm{BC}$ as well as $\mathrm{CE}$, mainly because it is the only farm animal species in Italy with a well-established monitoring system (European Commission Decision no. 2006/132/CE). Furthermore, bovine illegal slaughter is substantially absent.

Table 2. Characteristics of BC clusters.

\begin{tabular}{lccccc}
\hline Cluster ID & Radius $(\mathrm{m})$ & No. of farms included & No. of cases observed & No. of cases expected & P-value \\
\hline 1 & 2,210 & 61 & 9 & 0.50 & $<0.01$ \\
2 & 6,299 & 11 & 4 & 0.09 & 0.014 \\
\hline
\end{tabular}


Considering the low prevalence values $(3-10 \%)$ encountered in dogs in the hyper-endemic region of Sardinia using the sensitive polyclonal and monoclonal enzyme - linked immunosorbent assay (ELISA) systems (Varcasia et al., 2011), the chances to find positive animals with copromicroscopic survey in the definitive host in the hypo-endemic Veneto region is very low, unless the target survey population is accurately selected as it was in this study. It is worth noting that it is important to include dogs used for shepherding traditionally managed sheep flocks in (or passing through) cluster areas, since it has clearly been demonstrated that sheep breeding has a key-role in maintaining and spreading the disease (Scala et al., 2004b).

\section{Conclusion}

The prevalence of $\mathrm{CE}$ and $\mathrm{BC}$ is generally low in Europe with most areas hypo-endemic and therefore suitable for spatial analysis. Detection of bovine intermediate hosts represents the first step of a surveillance system aimed at the identification of major risk areas, where further epidemiological surveys for $\mathrm{CE}$ and $\mathrm{BC}$ in the definitive hosts (dogs and humans, respectively) should be implemented to break the parasite lifecycle and find the risk factors associated with these diseases.

For a survey system working on regular basis, the data flow from slaughterhouse inspection to ULSS veterinary services and on to a centralised office in charge of data gathering and elaboration should be improved through extension of existing electronic support systems and web-based communication, as implemented in other countries (Dupuy et al., 2013) and for other diseases (Yang et al., 2012; Porcasi et al., 2012).

\section{Acknowledgements}

This work was funded by the Veneto region (project DGRV 1222/2010).

\section{References}

Allepuz A, Gabriël S, Dorny P, Napp S, Jansen F, Vilar MJ, Vives L, Picart L, Ortuño A, Gutiérrez J et al., 2012. Comparison of bovine cysticercosis prevalence detected by antigen ELISA and visual inspection in the North East of Spain. Res Vet Sci 92, 393-395.

Allepuz A, Napp S, Picado A, Alba A, Panades J, Domingo M, Casal J, 2009. Descriptive and spatial epidemiology of bovine cysticercosis in North-Eastern Spain (Catalonia). Vet Parasitol $159,43-48$
Boone I, Thys E, Marcotty T, de Borchgrave J, Ducheyne E, Dorny, P, 2007. Distribution and risk factors of bovine cysticercosis in Belgian dairy and mixed herds. Prev Vet Med 82, $1-11$.

Brundu D, Aloi D, Rolesu S, Piseddu T, Masala G, 2012. Cystic echinococcosis in slaughtered cattle in Sardinia: a retrospective epidemiological study and spatial analysis. Geospat Health 6, 285-291.

Calvo-Artavia FF, Nielsen L, Dahl J, Clausen, D, Alban L, 2013. Occurrence and factors associated with bovine cysticercosis recorded in cattle at meat inspection in Denmark in 20042011. Prev Vet Med 110, 177-182.

Cringoli G, Rinaldi L, Musella V, Veneziano V, Maurelli MP, Di Pietro F, Frisiello M, Di Pietro S, 2007. Geo-referencing livestock farms as tool for studying cystic echinococcosis epidemiology in cattle and water buffaloes from southern Italy. Geospat Health 2, 105-111.

Davidson RK, Øines Ø, Madslien K, Mathis A, 2009. Echinococcus multilocularis - adaptation of a worm egg isolation procedure coupled with a multiplex PCR assay to carry out large-scale screening of red foxes (Vulpes vulpes) in Norway. Parasitol Res 104, 509-514.

Dupuy C, Morignat E, Maugey X, Vinard J, Hendrikx P, Ducrot C, Calavas D, Gay E, 2013. Defining syndromes using cattle meat inspection data for syndromic surveillance purposes: a statistical approach with the 2005-2010 data from ten French slaughterhouses. BMC Vet Res 9, 88.

Dwass M, 1957. Modified randomization tests for nonparametric hypotheses. Ann Math Stat 28, 181-187.

European Commission, 2000. Opinion of the scientific committee on veterinary measures relating to public health on the control of taeniasis/cysticercosis in man and animals. Available at: http://ec.europa.eu/food/fs/sc/scv/out36_en.pdf (accessed on November 2013).

Ferrè N, Mulatti P, Mazzucato M, Lorenzetto M, Trolese M, Pandolfo D, Vio P, Sitta G, Marangon S, 2011. GeoCREV: veterinary geographical information system and the development of a practical sub-national spatial data infrastructure. Geospat Health 5, 275-283.

Garippa G, Manfredi MT, 2009. Cystic echinococcosis in Europe and in Italy. Vet Res Commun 33, 35-39.

Guazzetti S, Micagni G, Ostanello F, Battelli G, 2006. Bovine echinococcosis in the province of Reggio Emilia (Italy): an example of integrated analysis of passive surveillance data. Parassitologia 48, 340.

Kulldorff M, 1997. A spatial scan statistic. Commun Stat Theory Methods 26, 1481-1496.

Kulldorff M, Nagarwalla N, 1995. Spatial disease clusters: detection and inference. Stat Med 14, 799-810.

Manfredi MT, Di Cerbo AR, Zanzani S, Moriggia A, Fattori D, Siboni A, Bonazza V, Felice C, Brunetti E, 2011. Prevalence of echinococcosis in humans, livestock and dogs in northern 
Italy. Helminthologia 48, 59-66.

Mastin A, Brouwer A, Fox M, Craig P, Guitián J, Li W, Stevens K, 2011. Spatial and temporal investigation of Echinococcus granulosus coproantigen prevalence in farm dogs in South Powys, Wales. Vet Parasitol 178, 100-107.

Pfeiffer DU, Robinson TP, Stevenson M, Stevens KB, Rogers DJ, Clements ACA, 2008. Spatial analysis in epidemiology. Oxford University Press, 142 pp.

Porcasi X, Rotela CH, Introini MV, Frutos N, Lanfri S, Peralta G, De Elia EA, Lanfri MA, Scavuzzo CM, 2012. An operative dengue risk stratification system in Argentina based on geospatial technology. Geospat Health 6, S31-S42.

Rinaldi L, Maurelli MP, Veneziano V, Capuano F, Perugini AG, Cringoli, S, 2008. The role of cattle in the epidemiology of Echinococcus granulosus in an endemic area of southern Italy. Parasitol Res 103, 175-9.

Scala A, Canu S, Tanda B, Basciu M, Polinas L, Sanna Coccone GN, Pilloni S, Canu S, Varcasia A, Garippa G, 2004a. An epidemiological and biomolecular survey of cystic echinococcosis in cattle in Sardinia. Parassitologia 46, 443-444.

Scala A, Varcasia A, Garippa G, 2004b. Cystic echinococcosis in Sardinia: the current role of sheep. Parassitologia 46, 397-400.

Trachsel D, Deplazes P, Mathis A, 2007. Identification of taeniid eggs in the faeces from carnivores based on multiplex PCR using targets in mitochondrial DNA. Parasitology 134, 911-920.

Umhang G, Richomme C, Boucher J, Hormaz V, Boué F, 2013. Prevalence survey and first molecular characterization of Echinococcus granulosus in France. Parasitol Res 112, 18091812.

Varcasia A, Tanda B, Giobbe M, Solinas C, Pipia AP, Malgor R, Carmona C, Garippa G, Scala A, 2011. Cystic echinococcosis in Sardinia: farmers' knowledge and dog infection in sheep farms. Vet Parasitol 181, 335-340.

Yang K, Sun L, Huang Y, Yang G, Wu F, Hang D, Li W, Zhang J, Liang Y, Zhou X, 2012. A real-time platform for monitoring schistosomiasis transmission supported by Google Earth and a web-based geographical information system. Geospat Health 6, 195-203. 